\title{
CREDIBILITY THEORY AND GENERALIZED LINEAR MODELS
}

\author{
J.A. NELDER ${ }^{1}$ and R.J. VERRALL ${ }^{2}$
}

\begin{abstract}
This paper shows how credibility theory can be encompassed within the theory of Hierarchical Generalized Linear Models. It is shown that credibility estimates are obtained by including random effects in the model. The framework of Hierarchical Generalized Linear Models allows a more extensive range of models to be used than straightforward credibility theory. The model fitting and testing procedures can be carried out using a standard statistical package. Thus, the paper contributes a further range of models which may be useful in a wide range of actuarial applications, including premium rating and claims reserving.
\end{abstract}

\section{KEYWORDS}

Credibility Theory; Hierarchical Generalized Linear Models; Generalized Linear Models; Premium Rating: Random-Effect Models.

\section{INTRODUCTION}

Credibility theory began with the papers by Mowbray (1914) and Whitney (1918). In those papers, the emphasis was on deriving a premium which was a balance between the experience of an individual risk and of a class of risks. Bühlmann (1967) showed how a credibility formula can be derived in a distribution-free way, using a leastsquares criterion. Since then, a number of papers have shown how this approach can be extended: see particularly Bühlmann and Straub (1970), Hachemeister (1975), de Vylder (1976, 1986). The survey by Goovaerts and Hoogstad (1987) provides an excellent introduction to these papers.

\footnotetext{
'Department of Mathematics

Imperial College

Huxley Buldıng

180 Queen's Gate

LONDON

SW7 2BZ

${ }^{2}$ Department of Actuarial Science and Statistics

City University

Northampton Square

LONDON

ECIV OHB

ASTIN BULLETIN Vol 27 No 11997 pp 7182
} 
The underlying assumption of credibility theory which sets it apart from formulae based on the individual risk alone is that the risk parameter is regarded as a random variable. This naturally leads to a Bayesian model, and there have been a large number of papers which adopt the Bayesian approach to credibility theory: for example Jewell (1974, 1975), Klugman (1987), Zehnwirth (1977). Klugman (1992) gives an introduction to the use of Bayesian methods, covering particularly aspects of credibility theory. A recent review of Bayesian methods in actuarial science and credibility theory is given by Makov et al (1996).

It can be shown that, under suitable assumptions, a credibility formula can be derived as the best linear approximation to the Bayesian estimate, using a quadratic loss function. Jewell (1974) showed that for an exponential family of distributions, the credibility formula is the same as the exact formula, so long as the conjugate prior distribution and a natural parametrisation is used. This result will be derived in a different way in section 3 , in order to place the basic model of credibility within a wider framework. The choice of structure for the collective and the parameterisation will be discussed in more detail. Since exponential families form the basis of Generalized Linear Models (GLMs) - see McCullagh and Nelder (1989) - it is natural to seek an extension of credibility theory encompassing the full range of models which can be formulated as GLMs. This is particularly apposite as GLMs have many very natural applications in the actuarial field: see, for example Renshaw (1991), Renshaw and Verrall (1994). This will also make possible more applications of credibility theory.

The main purpose of this paper is to show how credibility theory can be incorporated into the general framework of GLMs and implemented in the statistical package Genstat. Although the formulation of the credibility model is similar in many ways to the Bayesian approach, our approach is likelihood-based rather than Bayesian. The dispersion parameters will be estimated directly from the data without specifying prior distributions. No prior estimates for the parameters need to be supplied. All assumptions used in the model can be checked using, for example, appropriate residual analyses. Recent advances in the statistical literature on GLMs allow unobserved random effects to be estimated along with the parameter vector in the linear predictor. A useful recent paper is Breslow and Clayton (1993) which covers the theory of generalized linear mixed models (GLMMs). GLMMs allow the inclusion of normally distributed random effects and have been applied to a wide variety of statistical problems. We use the theory of Lee and Nelder (1996), which develops hierarchical generalized linear models (HGLMs). HGLMs also allow the inclusion of random effects, but these are not restricted to be normally distributed. Pure random-effect models, in which no fixed effects are included in the linear predictor, are known in the actuarial literature as credibility models. They form one part of a much wider class of models which have many potential applications to actuarial data.

Thus, the purpose of this paper is further to unify the actuarial theory; to show how modern statistical methods can be used to enable credibility theory to be applied in a standard statistical package; to allow extensions of basic credibility theory and to show how the assumptions made can be checked. This last point is important, since we 
regard many aspects of actuarial work as exercises is statistical modeling, rather than a dogmatic application of risk theory models.

It should be noted that the theory can be applied to models that specify only the mean and variance functions, using quasi-likelihood (Wedderburn, 1974, Nelder and Pregibon, 1987) - see section 5.

The paper is set out as follows. Section 2 contains a brief introduction to GLMs and derives some results which will be used elsewhere. Section 3 shows how credibility theory can be treated within the context of HGLMs. Section 4 outlines more general HGLMs, and how they are likely to be used for actuarial data. Section 5 outlines some extensions to the models in sections 3 and 4.

\section{INTRODUCTION TO GLMS}

This section contains a brief introduction to GLMs, and derives some of the key results which will be used later in the paper. A complete treatment of the theory and application of GLMs can be found in McCullagh and Nelder (1989).

The basis of GLMs is the assumption that the data are sampled from a oneparameter exponential family of distributions. We first describe these and some of their fundamental properties.

Consider a single observation $y$. A one-parameter exponential family of distributions has a log-likelihood of the form

$$
\frac{y \theta-b(\theta)}{\varphi}+c(y, \varphi)
$$

where $\theta$ is the canonical parameter

and $\varphi$ is the dispersion parameter, assumed known.

Haberman and Renshaw (1996) review the application of Generalized Linear Models in actuarial science, and include a section on loss distributions. In actuarial applications, many distributions belonging to one-parameter exponential families are useful. However, Haberman and Renshaw (1996) show how it is also possible to fit certain heavy-tailed distributions using Generalized Linear Models.

Some examples of such families are given below. It is straightforward to show that

$$
\begin{gathered}
\mu=E(Y)=\frac{d b(\theta)}{d \theta} \\
\text { and } \operatorname{Var}(Y)=\frac{d^{2} b(\theta)}{d \theta^{2}} \varphi .
\end{gathered}
$$

Note that $\operatorname{Var}(Y)$ is the product of two quantities. $\frac{d^{2} b(\theta)}{d \theta^{2}}$ is called the variance function and depends on the canonical parameter (and hence on the mean). We can write this as $V(\mu)$, since equation (2.2) shows that $\theta$ is a function of $\mu$. 


$$
\text { Thus } V(\mu)=\frac{d^{2} b(\theta)}{d \theta^{2}} \text {. }
$$

In actuarial applications, it is possible to include deterministic volume measures in the definition of $\operatorname{Var}(Y)$. A GLM may be defined by specifying a distribution, as above, together with a link function and a linear predictor. The link function defines the relationship between the linear predictor and the mean. The linear predictor takes the form

$$
\eta=X \beta
$$

where $\beta$ is parameter vector

and $\quad X$ is defined by the design.

For a single observation, $X$ is a row vector, and for a set of observations, $X$ is the design matrix.

The linear predictor is related to the mean by $\eta=g(\mu)$. The function $\mathrm{g}$ is called the link function, and the special case $g(\mu)=\theta$ is called the canonical link function.

By way of illustration, the log-likelihoods for some common distributions are given below.

(i) Normal

The log-likelihood is $\frac{\mu y-\frac{1}{2} \mu^{2}}{\sigma^{2}}-\frac{y^{2}}{2 \sigma^{2}}-\frac{1}{2} \log \left(2 \pi \sigma^{2}\right)$

Thus, $\theta=\mu$ and the canonical link function is the identity function.

$$
\begin{aligned}
& b(\theta)=\frac{\theta^{2}}{2} \text { and } c(y, \theta)=-\frac{y^{2}}{2 \sigma^{2}}-\frac{1}{2} \log \left(2 \pi \sigma^{2}\right) . \\
& V(\mu)=1 \text { and } \varphi=\sigma^{2} .
\end{aligned}
$$

(ii) Poisson

The log-likelihood is $y \log \mu-\mu-\log y$ !

$\theta=\log \mu$ and the canonical link is the $\log$ function.

$b(\theta)=e^{\theta}$ and $c(y, \varphi)=-\log y$ !

$V(\mu)=\mu$ and $\varphi=1$.

(iii) Binomial

Suppose $R \sim$ Binomial $(m, \mu)$. Define $Y=\frac{R}{m}$. Then the log-likelihood is

$$
\frac{y \log \frac{\mu}{1-\mu}-\log (1-\mu)}{\frac{1}{m}}+\log \left(\begin{array}{c}
m \\
m y
\end{array}\right) \text {. }
$$

Hence $\theta=\log \frac{\mu}{1-\mu}$, and the canonical link function is the logit function.

$$
b(\theta)=\log \left(1+e^{\theta}\right) \text { and } c(y, \varphi)=\log \left(\begin{array}{c}
m \\
m y
\end{array}\right) .
$$


$V(\mu)=\mu(1-\mu)$ and $\varphi=\frac{1}{m}$.

Note that this parameterisation may be unfamiliar because of the definition of $Y$. However, it enables us to give a coherent theory in the following section.

(iv) Gamma (with mean $\mu$ and variance $\frac{\mu^{2}}{v}$ ).

The log-likelihood is $\frac{-\frac{y}{\mu}+\log \frac{1}{\mu}}{\frac{1}{v}}+v \log y+v \log v-\log \Gamma(v)$.

$\theta=-\frac{1}{\mu}$ and the canonical link is the reciprocal function.

$b(\theta)=-\log (-\theta)$ and $c(y, \varphi)=v \log y+v \log v-\log \Gamma(v)$.

$V(\mu)=\mu^{2}$ and $\varphi=\nu^{-1}$.

This section has given a brief introduction to GLMs. The following section shows how standard credibility theory can be applied in this context. Section 4 will show how more general models can be formulated.

\section{THE BUHLMAN MODEL FOR EXPONENTIAL FAMILIES}

In this section, we derive the credibility formulae for exponential families of distributions, under the assumptions made by Bühlmann (1967). It is possible to extend this to other models: for example the assumptions of Bühlmann and Straub (1970) can be incorporated using weight functions. This section derives just the credibility formulae. A brief discussion of the estimation of the dispersion parameters is given in section 4 , where the appropriate references are cited.

Denote the data by $y_{l}$ for $i=1,2, \ldots, t ; j=1,2, \ldots, n_{t}$. Assume for the moment, as is common in credibility applications, that $n_{i}=k, \forall i$, but note that this restriction is not necessary for the derivation of HGLMs.

Thus, $i$ indexes the risks within the collective. In credibility theory, it is assumed that each risk has a risk parameter, which we denote by $\xi_{1}$ for risk $i$.

The assumptions of the model of Bühlmann (1967) are

(i) The risks, and hence $\xi_{1}$, are independently, identically distributed.

(ii) $y_{l j} \mid \xi_{1}$ are independently, identically distributed.

We assume that $y_{l j} \mid \xi_{l}$ is distributed according to an exponential family. Define $m\left(\xi_{l}\right)=E\left[y_{l j} \mid \xi_{l}\right]$. Note that under the assumptions of the model, $E\left[y_{l j} \mid \xi_{l}\right]$ does not 
depend on J. Hence the canonical parameter for observation $y_{i j}$ does not depend on $j$, and we assume that it can be written as follows.

$$
\theta_{l}^{\prime}=\theta\left(m\left(\xi_{l}\right)\right)=\theta\left(u_{l}\right)
$$

where $\theta$ is the canonical link function and $u_{t}$ is a random effect for group $i$. Thus, for the standard credibility model, $m\left(\xi_{t}\right)=u_{t}$. Define $v_{i}=\theta\left(u_{t}\right)$; then, in this case,

$$
\theta_{i}^{\prime}=v_{l} \text {. }
$$

Again, note that there is no $j$ dependence here. Note also that this also implies that $\operatorname{Var}\left(y_{\imath} \mid \xi_{\imath}\right)$ does not depend on $j$.

This has defined the distribution of the random variable within each risk, conditional on the risk parameter. It is also necessary to define the structure of the collective the distribution of $\left\{\xi_{\imath}: i=1, \ldots, t\right\}$. This is often done by defining a Bayesian prior distribution; here we use the same form of distribution for the random effects, but do not perform a Bayesian analysis. Instead, we define a "hierarchical likelihood", $h$, which we maximize.

We define the conjugate hierarchical generalized linear model (HGLM) by defining the kernel of the log-likelihood for $\theta\left(u_{\nu}\right)$ as

$$
a_{1} \theta_{t}^{\prime}-a_{2} b\left(\theta_{t}^{\prime}\right)
$$

In the actuarial literature, this distribution (the distribution of the random effects) is known as the structure of the collective. Note that we define the log-likelihood of $\xi$, implicitly through that of $\theta\left(m\left(\xi_{l}\right)\right)$. We have conditioned on $\xi_{t}$ through $m\left(\xi_{i}\right)=u_{l}$, since it is the latter that we wish to estimate.

From (3.3) and the distribution of $y_{l} \mid \xi_{1}$, we may define a hierarchical loglikelihood as

$$
\begin{aligned}
h & =\sum_{l, j} l\left(\theta_{i}^{\prime} ; y_{l} \mid v_{t}\right)+\sum_{l} l\left(v_{l}\right) \\
& =\sum_{l j}\left(\frac{y_{l} \theta_{i}^{\prime}-b\left(\theta_{l}^{\prime}\right)}{\varphi}\right)+c\left(y_{l}, \varphi\right)+a_{1} \theta_{i}^{\prime}-a_{2} b\left(\theta_{l}^{\prime}\right)
\end{aligned}
$$

When the distribution of both the data and the random effects is normal, this is Henderson's joint log-likelihood (Henderson 1975). In other cases, it is an obvious extension of the joint log-likelihood, called the hierarchical log-likelihood. We have now defined a hierarchical generalized linear model (HGLM), in this case the conjugate HGLM. In the particular case described in this section, the linear predictor for $y_{i j}$ consists solely of a random effects term which is modelled in the second stage of the likelihood, (3.2). It is possible to incorporate more structure into the model by including fixed effects and generalizing the form of the random effects model. However, in this section we are concerned solely with showing that the estimates obtained under the basic model described above are the usual credibility estimates. Thus, we require an estimate of $m\left(\xi_{i}\right)=u_{i}$. The mean random effects $\left\{u_{i}: i=1, \ldots, t\right\}$ are estimated by maximizing the hierarchical likelihood, (3.4), as follows. 
Using (2.2)

$$
\frac{\partial b\left(\theta\left(u_{t}\right)\right)}{\partial v_{t}}=u_{t}
$$

Hence

$$
\frac{\partial h}{\partial v_{t}}=\sum_{j=1}^{k}\left(\frac{y_{l J}-u_{t}}{\varphi}\right)+a_{1}-a_{2} u_{t}
$$

Equating $\frac{\partial h}{\partial v_{t}}$ to 0 gives

$$
y_{i+}-k \hat{u}_{t}+\varphi a_{1}-\varphi a_{2} \hat{u}_{1}=0
$$

where $y_{t+}=\sum_{j=1}^{k} y_{t j}$.

Hence

$$
\begin{aligned}
\hat{u}_{\imath} & =\frac{y_{l+}+\varphi a_{1}}{k+\varphi a_{2}} \\
& =Z \bar{y}_{l}+(1-Z) m
\end{aligned}
$$

where $\bar{y}_{l}=\frac{1}{k} y_{l^{+}}, \quad Z=\frac{k}{k+\varphi a_{2}} \quad$ and $m=\frac{a_{1}}{a_{2}}$.

Thus, we have shown that, with the choice of distribution for the random effects defined in (3.3), and using the canonical link function, the estimate of $u_{t}$ is in the form of a credibility estimate provided $E\left(m\left(\xi_{l}\right)\right)=\frac{a_{1}}{a_{2}}$. This is straightforward to show, and was also proved by Jewell (1974). The density of $u_{t}$ is proportional to

$$
\begin{aligned}
\frac{\partial e^{a_{1} \theta_{l}^{\prime}-a_{2} b\left(\theta_{l}^{\prime}\right)}}{\partial \theta_{l}^{\prime}} & =\left(a_{1}-a_{2} \frac{\partial b\left(\theta_{l}^{\prime}\right)}{\partial \theta_{l}^{\prime}}\right) e^{a_{1} \theta_{l}^{\prime}-a_{2} b\left(\theta_{i}^{\prime}-a_{2} b\left(\theta_{i}^{\prime}\right)\right.} \\
& =\left(a_{1}-a_{2} m\left(\xi_{l}\right)\right) e^{a_{1} \theta_{l}^{\prime}-a_{2} b\left(\theta_{l}^{\prime}\right)}
\end{aligned}
$$

Integrating over the natural range of $\theta_{l}^{\prime}$, and assuming $e^{a_{1} \theta_{i}^{\prime}-a_{2} b\left(\theta_{i}^{\prime}\right)}$ is zero at the end points, we have

$$
a_{1}-a_{2} E\left[m\left(\xi_{t}\right)\right]=0
$$

Hence, using (2.2),

$$
E\left[m\left(\xi_{t}\right)\right]=E\left[u_{t}\right]=\frac{a_{1}}{a_{2}} .
$$

Thus, we have shown that the credibility estimate is the same as the estimate obtained using a conjugate HGLM with pure random effects. This shows that credibility theory is closely connected to the statistical theory of random-effect models. Of course, it is possible to widen the scope of the models considerably. Fixed effects terms can also 
be included in the model, other link functions may be considered and the form of the random-effect models can be generalized.

It is possible to formulate the pure random-effect model in another way, by including a fixed effect which is constant for all the data. This means that the overall mean is estimated as a fixed effect and the randomeffects model departures from this overall mean. There is no effect on the credibility estimates, but the above derivation is, in some ways, closer to the actuarial theory.

The results in this section are closely related to those of Jewell (1974). The present approach differs in that it is not presented as a Bayesian procedure, and the emphasis is on the modelling aspects encapsulated within Generalized Linear Models.

The estimation of the dispersion parameters is discussed in section 4 . This includes the estimation of $\varphi$ and of $a_{1}$ and $a_{2}$. It should be noted that if a constant fixed effect is included in the model, as outlined above, there is only one parameter to estimate in the distribution of $u_{\imath}$. For this reason we adopt this approach henceforth.

By way of illustration, we consider the four exponential families outlined in section 2. Note that we can derive the density of $u_{t}$ from the density of $\theta\left(u_{t}\right)$, defined in (3.3). The density of $u_{i}$ is proportional to

(ii) Normal

$$
\begin{gathered}
e^{a_{1} \theta_{\imath}^{\prime}-a_{2} b\left(\theta_{i}^{\prime}\right)} \frac{\partial \theta\left(u_{\imath}\right)}{\partial u_{\iota}} \\
\frac{e^{a_{1} \theta_{\imath}^{\prime}-a_{2} b\left(\theta_{\imath}^{\prime}\right)}}{V\left(u_{\imath}\right)}
\end{gathered}
$$

The random effects have log-likelihood whose kernel is

$a_{1} u_{l}-a_{2} \frac{u_{i}^{2}}{2}$

i.e. $u_{i} \sim N\left(m, \sigma_{0}^{2}\right) \quad a_{1}=\frac{m}{\sigma_{0}^{2}}, a_{2}=\frac{1}{\sigma_{0}^{2}}$ and $m=E\left[u_{l}\right]=\frac{a_{1}}{a_{2}}$

(ii) Poisson

$u_{i}$ has a likelihood proportional to

$e^{a_{1} \log u_{i}-a_{2} u_{i}}$

$u_{t}$

Hence $u_{t} \sim$ Gamma, parameters $a_{1}$ and $a_{2}$, and $m=E\left[u_{l}\right]=\frac{a_{1}}{a_{2}}$.

(iii) Binomial

$u_{1}$ has a likelihood proportional to

$\exp \left[a_{1} \log \left(\frac{u_{t}}{1-u_{t}}\right)-a_{2} \log \left(\frac{1}{1-u_{t}}\right)\right]$

$u_{l}\left(1-u_{l}\right)$

i.e. $u_{t} \sim$ Beta, parameters $a_{l}$ and $a_{2}-a_{l}$, and $m=E\left[u_{l}\right]=\frac{a_{1}}{a_{2}}$. 
(iv) Gamma

$u_{i}$ has a likelihood proportional to

$\frac{\exp \left(\frac{-a_{1}}{u_{t}}+a_{2} \log u_{t}\right)}{u_{t}^{2}}$

i.e. $u_{t} \sim$ inverse gamma and $m=E\left[u_{l}\right]=\frac{a_{1}}{a_{2}}$.

Having shown that the estimates obtained using conjugate HGLMs for a simple random-effect model are the usual credibility estimates, we now define a more general framework which encompasses credibility models.

\section{HIERARCHICAL GENERALIZED LINEAR MODELS}

Standard GLMs model differences between groups, parametric variation and other effects as fixed effects in the linear predictor. Random-effect models can be combined with standard GLMs in order to formulate models with both fixed effects and the random effects of credibility models. To do this, we define an extended linear predictor for a single observation as

where $\eta=X \beta$, as in (2.5)

$$
\eta^{\prime}=\eta+v
$$

and $\quad v$ is a strictly monotonic function of $u, v=v(u)$.

When $v=0,(4.1)$ reduces to the standard linear predictor for GLMs. When $\eta=0$ and $v=\theta(u)$, we have the basis credibility model described in section 3 .

The hierarchical log-likelihood, (3.4), becomes

$$
h=\sum_{i, j} l\left(\beta, y_{i j} \mid v_{i}\right)+\sum_{i} l\left(v_{i}\right)
$$

where $v_{t}=v\left(u_{t}\right)$.

The maximum hierarchical likelihood extimates (MHLEs) of $\beta$ and $u$ are obtained from the pair of equations

$$
\frac{\partial h}{\partial \beta}=0 \text { and } \frac{\partial h}{\partial v}=0
$$

which may be solved iteratively using the procedures written by the second author for the statistical package Genstat.

We consider here the case when the canonical link function is used for the fixed effects and $v=\theta(\mathrm{u})$. In this case, equation (3.1) for observation $y_{i j}$ becomes

$$
\theta_{y}^{\prime}=\theta_{l}+\theta\left(u_{l}\right)
$$

where $\theta_{l}=X_{\imath} \beta$

$\theta$ is the canonical link function 
and $X_{u}$ is the row from the design matrix for the fixed effects which relates to $y_{t}$.

The same log-likelihood is used for $\theta\left(u_{\nu}\right)$, as in (3.3). Then the kernel of $h$ is

Hence

$$
\frac{\sum_{l, J}\left(y_{i j} \theta_{t j}^{\prime}-b\left(\theta_{t j}^{\prime}\right)\right)}{\varphi}+\sum_{i} l\left(v_{t}\right) .
$$

$$
\frac{\partial h}{\partial \beta_{k}}=\frac{\left.\sum_{l, j}\left(y_{l j}-u_{l j}^{\prime}\right)\right) x_{k l}}{\varphi}
$$

and

$$
\frac{\partial h}{\partial v_{l}}=\frac{\left.\sum_{l, j}\left(y_{l j}-u_{l j}^{\prime}\right)\right)+\varphi a_{1}}{\varphi}-a_{2} u_{t}
$$

where $u_{i j}^{\prime}=E\left[y_{i j} \mid u_{i}\right]=E\left[y_{i j} \mid \xi_{i}\right]$,

$\beta_{k}$ is the $k$ th parameter in the fixed effects

and $\quad x_{k l}$ is the kth entry of the row vector $X_{l j}$.

Note that in this case, unlike that in section $3, E\left[y_{l y} \mid \xi_{l}\right] \neq u_{l}$. Instead,

$$
\theta\left(u_{i j}^{\prime}\right)=\eta_{t j}+\theta\left(u_{t}\right)
$$

which implies that $\mu_{l j}^{\prime}=u_{\imath}$ when $\eta_{i j}=0$.

We include the overall mean as a fixed effect and require that the random effects then have the appropriate mean (eg 0 for the identity link function).

The dispersion parameters given the fixed and random effects are estimated by maximising the h-likelihood after a suitable adjustment. The adjustment, which results in an adjusted profile $\mathrm{h}$-likelihood, is necessary because the marginal maximum likelihood estimates may be biased. Further justifications for this adjustment can be found in Cox and Reid (1987) and Lee . nd Nelder (1996). For the normal distribution, unbiased estimates are obtained. Morc details on estimation theory for random-effect GLMs can be found in McGilchrist (1994) and Schall (1991).

The joint estimates of the mean effects (fixed and random) and the dispersion parameters are obtained by iterating between the two sets of estimating equations. These processes may be conveniently carried out in Genstat, for which a set of procedures is available from the second author.

For the distributions illustrated in section 1, the likelihoods of the random effects are again appropriate, but the estimate will be different because of the difference between (3.1) and (4.2). 


\section{DISCUSSION}

It is possible to extend the class of models to which these methods may be applied by specifying just the mean and variance functions. This is useful when greater flexibility is required in the modelling assumptions. For example, Renshaw and Verrall (1994) show that the chain-ladder technique in claims reserving is essentially equivalent to GLM with a Poisson likelihood and an appropriate linear predictor. By specifying just the mean and variance function, this model may be applied to a much wider class of data than is implied by the Poisson assumption (which obviously requires the variance to equal the nean). This involves the use of extended quasi-likelihood (Wedderburn 1974, Nelder and Pregibon 1987). For HGLMs, the equivalent extension is the extended quasi-h-likelihood, in which the extended quasi-likelihood is used in the hierarchical likelihood. This extension makes it possible, for example, to include random effects in the chain-ladder linear model to allow a connection between accident years.

HGLMs may also be of use when a particular factor is hard to model parametrically. An example of this, which has been mentioned above, is claims reserving, when it is inappropriate to model the accident years as completely independent, but a parametric relationship is also inappropriate. The same comment applies in motor premium rating, when it is usual to group a factor such as the age of the policyholder. Such a grouping may be inappropriate, as it may be crude or doubtful because it has been decided before the analysis of the data (for example, according to the present rating structure). However, it is often inappropriate, because of computational and theroretical considerations, to treat the ages as completely separate or to apply a parametric model. In this situation, HGLMs may be useful.

Applications in life insurance include similar premium-rating situations as in general insurance, and also graduation theory. The use of HGLMs for graduation would have some similarities to Whittaker graduation, which can be regarded as a GLM with a stochastic linear predictor (Verrall, 1993).

\section{REFERENCES}

Breslow, N.E. and Clayton D.G. (1993) Approximate inference in generalised linear mixed models. $J$. Am. Statist. Ass., 88, 9-25.

Bühlmann, H. (1967) Experience Rating and Credibility. ASTIN Bulletin, 4, 199-207.

Bühlmann, H. and Straub, E. (1970) Credibility for Loss Ratios. ARCH, 1972.2.

Cox, D.R. and REID, N. (1987) Parameter orthogonality and approximate conditional inference. J.R. Statist. Soc, B., 49, 1-39

Goovaerts, M. and Hoogstad, W. (1987) Credibility Theory. Surveys of Actuarial Studies No. 4, Rotterdam: Nationale-Nederlanden.

Haberman, S. and Renshaw, A.E., (1996) Generalized Linear Models and actuarial science. The Statistician, $\mathbf{4 5}, 407-436$.

HACHEMEISTER, C.R. (1975) Credibility for Regression Models with Applications to Trend, in Credibility: Theory and Applications, P. Kalm, ed., New York: Academic Press.

Henderson, C.R. (1975) Best linear unbiased estimation and prediction under a selection model. Biometrics, 31, 423-447.

JEWELl, W.S. (1974) Credible means are exact Bayesian for exponential families. ASTIN Bulletin, 8, 77-90.

JEWELL, W.S. (1975) The use of collateral data in credibility theory: a hierarchical model. Giornale dell'Instituto Italiano degli Attuari, 38, 1-16. 
Klugman, S. (1987) Credibility for Classification Ratemaking via the Hierarchical Normal Linear Model. Proc. of the Casualty Act. Soc., 74, 272-321.

Klugman, S. (1992) Bayesian Statistics in Actuarial Science with Emphasis on Credibility. Kluwer Academic Publishers.

LeE Y. and Nelder, J.A. (1996) Hierarchical Generalized Linear Models. J.R. Statist. Soc. B, 58, 619-678.

MCCullagh, P. and Nelder, J.A. (1989) Generalized Linear Models. 2nd Edition. London: Chapman and Hall.

MCGILCHRIST, C.A. (1994) Estimation in generalized mixed models. J.R. Statist. Soc. B, 56, 61-69.

MaKov, U.E., SMith, A.F.M. and LiU, Y.-H. Bayesian methods in actuarial science. The Statistician, 45, 503-515.

MowBray, A.H. (1914) How extensive a payroll exposure is necessary to give a dependable pure premium. Proc. of the Casualty Act. Soc., 1, 24-30.

Nelder, J.A. and LeE, Y. (1992) Likelihood, quasi-likelihood and pseudo-likelihood: Some coomparisons. J.R. Statist. Soc. B, 54, 273-284.

Nelder, J.A. and Pregibon,D. (1987) An extended quasi-likelihood function. Biometrika, 74, 221 -231.

Renshaw A.E. (1991) Actuarial Graduation Practice and Generalized Linear and Non-Linear Models. $J$. Inst. Acts., 118, 295-312.

Renshaw, A.E. and Verrall, R.J. (1994) A stochastic model underlying the chain-ladder technique, Proceedings, ASTIN Colloquium, 1994.

SCHALL, R. (1991) Estimating in generalized linear models with random effects. Biometrika, 78, 719-727.

VERRALL, R.J. (1993) A state space formulation of Whittaker graduation, with extensions. Insurance: Mathematics and Economics, 13, 7-14.

DE VYLDER, F. (1976) Optimal semilinear credibility. Bull. of the Assoc. of Swiss Acts., 78, 27-40.

DE VYLDER, F. (1986) General regression in multidimensional credibility theory, in Insurance and Risk Theory, ed. M. GovaerTs, J; HAEZENDONCK and F. DE VYLDER, Reidel.

WEDDERBURN, R.W.M. (1974) Quasi-likelihood functions, generalized linear models and the Gauss-Newton method. Biometrika, 61, 439-447.

Whitney, A.W. (1918) The theory of experience rating. Proc. of the Casualty Act. Soc., 4, 274-292.

ZEHNWIRTH, B. (1977) The mean credibility formula is a Bayes rule. Scandinavian Actuarial Journal, 4, $212-216$ 